Michael Frank*

\title{
Bibliotheksinformatik als Studienrichtung an der HTWK Leipzig
}

https://doi.org/10.1515/bfp-2019-2065

Zusammenfassung: Moderne Bibliotheken brauchen geeignete Informationssysteme, um digitale Wissensquellen dem Publikum zugänglich zu machen. Ein relativ neues Tätigkeitsfeld ist das Forschungsdatenmanagement, das der Beschreibung von Kontexten von Forschungsergebnissen dient. Um derartige Informationssysteme zu schaffen, braucht man Spezialisten mit einer dualen Ausbildung in Bibliothekswissenschaft und Informatik. An der HTWK Leipzig wird ein derartiges Bildungsangebot erprobt, um Bibliotheksinformatiker auszubilden. Der vorliegende Artikel gibt einen Einblick in die Ideen, die zu dieser Entwicklung führten.

Schlüsselwörter: Informationssysteme; Forschungsdatenmanagement; Bibliotheksinformatiker; spezialisierte Ausbildung an der HTWK Leipzig

\section{Digital Library Science as an Educational Offer at HTWK Leipzig}

\begin{abstract}
Modern libraries depend on suitable information systems to make digital knowledge sources available to the public. One relatively new goal is the management of research data describing the context of research results. To maintain such information systems specialists with a dual education in both library science and computer science are needed. Such a special education opportunity has been established at HTWK Leipzig to educate digital library specialists. The present paper gives an insight into the ideas governing this development.
\end{abstract}

Keywords: Information systems; research data management; digital librarians; specialized education at HTWK Leipzig

\section{Rahmenbedingungen}

Das Bibliotheks- und Publikationswesen und die Formen der wissenschaftlichen Kommunikation sind relativ stabile Branchen in der Gesellschaft, die einen anhaltend hohen

*Kontaktperson: Michael Frank, michael.frank@htwk-leipzig.de
Bedarf an Wissensbewahrung und -vermittlung hat. Der Bedarf an geprüften, zitierfähigen Informationen in persistenten Quellen und der schnelle selektive Zugang zu ihnen sind groß, er wird aber auch von sich ausweitenden Tendenzen des interessensgetriebenen Verzichts auf Fundierung von verbreiteten Behauptungen konterkariert. Der gesellschaftliche Prozess der zunehmend digitalen Wissenserstellung, Wissensverwaltung und Wissensnutzung stellt die traditionellen Institutionen vor die Aufgabe, das Selbstverständnis und die Geschäftsprozesse dahingehend zu verändern, immer mehr digitale Inhalte in mannigfaltigen Datenformaten aus unterschiedlichen Quellen und in unterschiedlichen Rechtsformen vernetzt der Gesellschaft anbieten $\mathrm{zu}$ können - und zu müssen. Gleichzeitig muss der dadurch verursachten relativen Unbeständigkeit von Wissen und der wachsenden Komplexität seiner inneren Vernetzung entgegengewirkt werden. Neu erwachsen Möglichkeiten, Metadaten und Medien digital miteinander zu verbinden und über Benutzungsinstanzen hinweg verbunden zu transportieren. Neben den verschiedensten digitalen Archiven und Medienspeichern in unterschiedlicher Rechtsträgerschaft und Rechtsform entstehen leistungsfähige Informationssysteme, die von den bibliothekarischen Institutionen zu nutzen und mit weiterzuentwickeln sind. In der Arbeitsweise der Bibliothekare selbst muss eine starke Informationskompetenz auf den Interessensfeldern der jeweiligen Bibliotheksnutzer erworben werden, um aus bibliothekarischen und öffentlichen Quellen in Konkurrenz zu anderen Informationsanbietern für den Benutzer attraktive Wissensaggregationen anzubieten und dadurch die tradierte starke Stellung in der Wissenswirtschaft zu bewahren. Dabei sind auch Geschäftsmodelle zu überdenken.

Ein relativ neu zu erschließendes Geschäftsfeld ist das Forschungsdatenmanagement. Es geht darum, Daten und Informationssammlungen im Vorfeld und im Kontext von Forschungsprojekten zu erhalten und der Nutzung zusammen mit den daraus erwachsenen Publikationen zu erschließen. Für die Natur- und Ingenieurwissenschaften ist die Nachvollziehbarkeit von Forschungsergebnissen besonders bei Verwendung von empirisch erhobenen Daten schon lange eine wissenschaftsimmanente Forderung. In den verschiedenen Wissenschaftsfeldern haben diese Daten sehr unterschiedliche mediale bzw. digitale Formen, Datenstrukturen und Zusammensetzungen, auch die Da- 
tenumfänge sind in verschiedenen infrage kommenden Forschungsfeldern in Größenordnungen verschieden (z. B. sehr große Datenmengen in der Computertomografie oder in bildgestützten Weltraumprojekten). Informationswissenschaftliche Institutionen wollen sich auf das Planen und Strukturieren, auf die tägliche Arbeit mit und an Forschungsdaten, auf Einhaltung von Rechten und Pflichten in diesen Prozessen, auf das Aufbereiten und Veröffentlichen und natürlich auf die Bereithaltung zur Nachnutzung konzentrieren. Im Idealfall soll eine Umgebung zur Begleitung und Dokumentierung von konkreten Forschungsprojekten entstehen, die nach Abschluss der Forschungsprojekte zur Bewahrung des Forschungskontextes sowie der Forschungsergebnisse führt und deren Nachnutzung bzw. Erweiterung ermöglicht. ${ }^{1}$ Dieses Tätigkeitsfeld ist noch stark in der Entwicklung und Formierung begriffen, so dass eine abschließende Beschreibung nicht gegeben werden kann.

Für die sich abzeichnenden neuen Aufgaben im Zuge der Digitalisierung der Wissenswirtschaft und des Forschungsdatenmanagements besteht eine zunehmende Nachfrage nach Absolventen mit Qualifikationen sowohl in den Informationswissenschaften als auch in der Informatik, die kundenorientiert neue digitale Systeme und Anwendungsfälle schaffen können. Zu deren Ausbildung leistet die Studienrichtung Bibliotheksinformatik an der HTWK Leipzig einen Beitrag.

\section{Hintergründe der Einrichtung der Studienrichtung}

Die genannten Tendenzen in der Arbeitsweise von Bibliotheken, von Fachinformationsdiensten oder von Unternehmen der Wissenswirtschaft führten an der HTWK Leipzig 2011-2012 zu Gesprächen zwischen Informationswissenschaftlern und Informatikern, wie mit den vorhandenen Ressourcen in der Lehre Spezialisten zur weiteren Entwicklung der Infrastruktur und der Prozesse im Bibliothekswesen und in den Informationswissenschaften ausgebildet werden könnten. Die Voraussetzungen waren an der HTWK Leipzig mit langjährig entwickelten konsekutiven Studiengängen in Bibliotheks- und Informationswissenschaften und in Informatik bzw. Medieninformatik sehr gut gegeben. Beide Bereiche waren von einer langjährig gewachsenen Fachkultur in den Kollegenkreisen geprägt, die von einer Offenheit für neue Entwicklungen getragen war.

1 forschungsdaten.info (2019), forschungsdaten.org (2019).
In der Tendenz fiel die Entscheidung für eine spezialisierte Informatikausbildung, in die Standards des Bibliothekswesens und der Informationswissenschaften in einer frühen Studienphase stark integriert werden. Die Ausbildung sollte kooperativ zwischen den beiden Fachgruppen unter Nutzung der vorhandenen Lehrveranstaltungen in den beiden beteiligten Bachelorstudiengängen Medieninformatik und Bibliotheks- und Informationswissenschaften erfolgen. Mit diesem Ansatz wird das Denken der Studierenden auf semantische Aspekte der Anwendungssphären der eingesetzten Technologien gelenkt, die in der Informatik als eher mathematisch-algorithmisch orientierter Disziplin nur langsam in Lösungen Eingang finden. Das führt bei den Studierenden zu einer anderen Wahrnehmung der Studieninhalte und zu anderen Setzungen von Prioritäten in der Ausprägung der Spezialisierung. Die Realisierung der Idee war eine Studienrichtung im Studiengang Medieninformatik Bachelor, zu deren Gestaltung etwa $30 \%$ der Lehrinhalte des vorhandenen Studiengangs Bibliotheks- und Informationswissenschaften Bachelor und etwa $70 \%$ der Lehrinhalte des vorhandenen Studiengangs Medieninformatik Bachelor durch Besuch vorhandener Lehrveranstaltungen herangezogen wurden. Als Bezeichnung wurde der Begriff Bibliotheksinformatik gewählt, um den Charakter einer breiten Grundlagenausbildung in Informatik mit der Fokussierung auf die Anwendungssphäre der Bibliotheken, Fachinformationsdienste und Unternehmen der Wissenswirtschaft, auf die Bedürfnisse der Benutzer und letztendlich auf das praktische Wirken in der Gesellschaft zu betonen. Seit 2013 werden nun relativ wenige, aber sehr gut motivierte Studierende immatrikuliert und ausgebildet. Als Fachhochschule kann die HTWK Leipzig auch Menschen mit Berufsausbildung und Fachhochschulreife zum Studium aufnehmen. Die Ausbildung ist stark praxisorientiert, u. a. mit einem Informationspraktikum im zweiten Fachsemester, einem zwölfwöchigen Pflichtpraktikum in Einrichtungen gemäß der Studienorientierung im fünften Fachsemester und meist mit einer anschließenden kooperativ gestalteten Graduierungsphase von drei bis fünf Monaten Dauer zum Studienabschluss. Im Anschluss an das Bachelorstudium kann in verschiedene Masterstudiengänge gewechselt werden. Die Ordnungen können unter HTWK (2013-2019) nachgelesen werden.

\section{Studienaufbau, Studieninhalte}

Im Grundstudium der Bachelorstudiengänge Informatik und Medieninformatik werden viele grundlegende Informatikmodule für beide Studiengänge gemeinsam abgehal- 
ten. Das betrifft u. a. Mathematik, Modellierung als praxisorientierte Einführung theoretischer Grundkonzepte der Informatik, das Erlernen zweier vollständiger Programmiersprachen (Java und C\#), Algorithmen und Datenstrukturen, Betriebssysteme und Rechnernetze, Datenbanken und Softwaretechnik. Es gibt ein großes Softwarepraktikum in Gruppen, in dem die planvolle Entwicklung einer Softwarelösung in rollenbasierter Arbeitsteilung mit simulierten Auftraggebern und Masterstudierenden als Projektleitern trainiert wird. Medieninformatik- und Bibliotheksinformatikstudierende haben einige von der Ausbildung der Informatiker abweichende Module, an Stelle von vertiefter Mathematikausbildung, Hardwarepraktikum, Digitaltechnik und theoretischer Informatik. So gibt es für beide Gruppen zwei Multimedia-Grundkurse. Insbesondere besuchen die angehenden Bibliotheksinformatiker im Studiengang Bibliotheks- und Informationswissenschaft Bachelor Module wie Einführung in die Bibliotheks- und Informationswissenschaften, Informationsvermittlung und Bibliographie, IT-gestützte Informationsbereitstellung durch Bibliotheken, Inhaltserschließung, Medienerschließung und Formalerschließung. Mit dieser grundlegenden Orientierung im anderen Fachgebiet erfolgt im anschließenden Hauptstudium gemeinsam mit allen Medieninformatikern die vertiefte Ausbildung in frontendorientierter Informatik, wie z. B. durch Module in Multimedialer Webprogrammierung, IT-Sicherheit, MultimediaDatenbanken, XML-gestützten Datenverarbeitungsprozessen und Datenbanken II. Im vierten bzw. fünften Fachsemester des Studiums müssen drei Wahlpflichtmodule im Bereich beider Ausbildungsrichtungen belegt werden, so dass je nach persönlichem Werdegang und Berufsziel Zusatzqualifikationen erworben werden können.

Im sechsten Fachsemester schließt sich das mindestens zwölfwöchige Betriebspraktikum an. In Leipzig bestehen mit den Informatikgruppen direkt an der Universitätsbibliothek (Digitale Dienste, Digitale Sammlungen) und am Universitätsrechenzentrum der Universität Leipzig (Informationssysteme zu diversen digitalen Archiven) zwei sehr interessante Partnereinrichtungen, die gern Praktikanten aus der Studienrichtung Bibliotheksinformatik beschäftigen. Dabei werden die Praktikanten in echte Projekte eingebunden und erhalten somit auch Anregungen für die anschließende Phase des Erarbeitens der Bachelorarbeit im Kontext der Arbeitsgruppen. ${ }^{2}$ Andere Orte für Praktika bestehen in Leipzig mit der Stadtbibliothek, dem Leipziger Standort der Deutschen Nationalbibliothek, einer Filiale des Sächsischen Staatsarchivs und zahlreichen

2 Vgl. Kupferschmidt (2016), Seige (2017).
Fachbibliotheken in den Max-Planck-, Helmholtz-, Fraunhofer-Instituten und Instituten der Leibniz-Gemeinschaft der Stadt. Auch das Universitätsklinikum war schon einmal Beschäftigungsort. Zudem ist mit der Martin-LutherUniversität zu Halle/S. eine weitere größere Universität in Reichweite.

\section{Einsatzgebiete der Absolventen der Studienrichtung}

Absolventen der Studienrichtung Bibliotheksinformatik im Studiengang Medieninformatik an der HTWK Leipzig sind insbesondere befähigt, an der Gestaltung der Interaktionsoberflächen, an der Ausgestaltung der programmierten Angebote für Benutzer und der Datenbasis großer Informationssysteme kundenorientiert mit Branchenwissen mitzuwirken. Auch die Auswahl bzw. Neugestaltung von Metadatensystemen zur systematisierten Verwaltung der Daten liegen in ihrer Kompetenz. Das betrifft auch Aspekte des Forschungsdatenmanagements, soweit es deren Verwaltung, Systematisierung und Sichtung anbelangt. Um die interaktive, analytische Auswertung von naturwissenschaftlichen Forschungsdaten zu unterstützen, ist eine Vertiefung der Ausbildung in Informationsvisualisierung und Computergrafik, in Mathematik und Künstlicher Intelligenz anzustreben. Das sprengt die zeitlichen Möglichkeiten einer Bachelorausbildung und ist nur mit einem Masterstudium in Informatik oder Medieninformatik zu adressieren, was als mögliche Folgeausbildung an der HTWK Leipzig angeboten wird. Ziel wäre es, Werkzeuge im Sinne von Visual Analytics zu schaffen, um je nach Natur der Forschungsdaten zusätzlich zur reinen Sichtung eine explorative und hypothesenbildende Analyse der Daten anbieten zu können und die Auswahl der längerfristig aufzubewahrenden Daten fachlich begründet durchzuführen.

\section{Literaturverzeichnis}

Hochschule für Technik, Wirtschaft und Kultur (HTWK) Leipzig, Studiengang Medieninformatik, Studienrichtung Bibliotheksinformatik (2013-2019): Beschreibung und Ordnungen. Verfügbar unter https://www.htwk-leipzig.de/studieren/studiengaenge/b achelorstudiengaenge/medieninformatik/medieninformatik-stu dienrichtung-bibliotheksinformatik.

forschungsdaten.info (2019): Forschung und Daten managen. Verfügbar unter https://www.forschungsdaten.info.

forschungsdaten.org (2019): Wiki und Informationssammlung zu DFG-Projekten rund um den Umgang mit digitalen Forschungsdaten. Verfügbar unter https://www.forschungsdaten.org. 
Kupferschmidt, Jens (2016): Die Entwicklungsgeschichte von MyCoRe an der Uni Leipzig. Digital Classics Online, 2 (2), 30-34. Verfügbar unter https://journals.ub.uni-heidelberg.de/index.ph $\mathrm{p} / \mathrm{dco} /$ article/viewFile/32387/26137.

Seige, Leander (2017): IIIF - Kulturinstitutionen schaffen interoperable Schnittstellen für digitalisiertes Kulturgut. Leipzig, 34C3. Verfügbar unter https://ftp.fau.de/cdn.media.ccc.de/congress/ 2017/slides-pdf/34c3-8853-international_image_interoperabil ity_framework_iiif_kulturinstitutionen_schaffen_interoperable_ schnittstellen_fur_digitalisiertes_kulturgut.pdf.

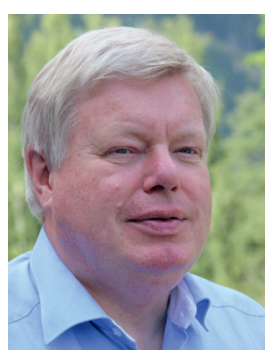

Michael Frank

Hochschule für Technik, Wirtschaft und Kultur Leipzig

Fakultät Informatik und Medien

PF 301166

D-04251 Leipzig

michael.frank@htwk-leipzig.de 